\title{
INTER-DIVISION IV-V / WORKING GROUP ACTIVE B-TYPE STARS
}

\author{
CO-CHAIRS \\ PAST CHAIR \\ MEMBERS
}

\author{
Juan Fabregat, Geraldine J. Peters \\ Stanley P. Owocki \\ Karen S. Bjorkman, Douglas R. Gies, \\ Hubertus F. Henrichs, David A. McDavid, \\ Coralie Neiner, Philippe Stee
}

\section{TRIENNIAL REPORT 2006 - 2009}

\section{Introduction}

The Working Group on Active B-type Stars (formerly known as the Working Group on Be Stars) was re-established under IAU Commission 29 at the IAU General Assembly in Montreal, Quebec (Canada) in 1979, and has been continuously active to the present. Its main goal is to promote and stimulate research and international collaboration on the field of the active early-type (OB) stars.

The interest of the WG were originally focused on classical Be stars research. The recent years have seen a broadening of the group interests, with an increasing contact and overlap with other research areas, particularly for closely aligned topics like pulsating OB stars.

\section{Developments within the past triennium}

The Be Star Newsletter (see <www. astro.virginia.edu/ dam3ma/benews/>) has continued to be the main source of information on new discoveries, ideas, manuscripts, and meetings on active B stars. G. Peters, D. Gies, and D. McDavid continue, respectively, as Editor-in-Chief, Technical Editor, and Web master. The issue No. 38 was published in March 2007, and contains, among other items, 18 notes and advertisements of interest for the active B stars community and 36 abstracts of papers relevant to its research subjects. The current working issue No. 39 contains 6 research notes and 18 abstracts as by June 30th.

The proceedings of the last major meeting on active B stars, Active OB-Stars: Laboratories for Stellar and Circumstellar Physics, held in Sapporo (Japan), were published in March 2007 (Okazaki, Owocki \& Stefl 2007).

In general grounds, the field of the Be star research has been very active during the last few years, and we expect this activity to increase. The recent development of powerful observational equipment has led to significant progress in our knowledge of active B stars physics. The advent of the VLTI interferometer and the CHARA Array have allowed the spatial resolution of several Be star photospheres and disks (e.g., Domiciano de Souza et al. 2003; Gies et al. 2007), and the finding of the Keplerian rotation in the disk around $\alpha$ Arae (Meilland et al. 2007) . The application of spectropolarimetric techniques resulted in the discovery of magnetic fields in a few Be stars (Neiner et al. 2003), and the 
commissioning of new and more powerful spectropolarimeters will allow the systematic study of the prevalence of magnetic fields among Be stars and their relation with the mass ejection episodes.

Perhaps the most exciting current development is the accurate photometric monitoring of Be stars from outside our atmosphere. The Canadian space mission MOST, launched on 2003 June 30, has already provided photometric time series of unprecedented quality of several bright Be stars, revealing a rich spectrum of frequencies associated with radial and nonradial pulsations (Walker et al. 2005a, 2005b; Saio et al. 2007; Cameron et al. 2008). The French led CoRoT satellite was successfully launched on December 2006, and started its scientific observations on 3 February 2007. CoRoT is providing photometry of many B and Be stars in continuous runs lasting from 20 to 150 days. The analysis of the photometric time series and the interpretation of the results with the techniques of asteroseismology will provide important insights on the internal structure of Be stars, the role of nonradial pulsations, and its relation with the Be star outbursts (Michel et al. 2006).

Although opportunities to study Be stars in the FUV are currently limited, new spectra were recently obtained of Be stars in our Galaxy and the Magellanic Clouds with the FUSE spacecraft. This new data will provide information on the abundances of carbon, nitrogen, and the iron group elements in the photospheres of Be stars that will complement and serve as a check on the results and predictions from the planned asteroseismology investigations as well as theories for Be stars based upon the concept of critical rotation.

The rapid rotation of Be stars may be caused in some cases by past mass and angular momentum accretion in an interacting binary. The mass donor would currently appear as a hot subdwarf, stripped of its outer envelope and N-rich as the CNO-processed core is now exposed. Recently the presence of a subdwarf companion to the Be star FY CMa was detected (Peters et al. 2008) and thus this bright Be star becomes the second confirmed example, after $\phi$ Per, of a $\mathrm{Be}+\mathrm{sdO}$ system.

Other important tools that will be available for Be star researchers in the near future are the ongoing and planned deep photometric galactic plane surveys. In the northern hemisphere the $\mathrm{H} \alpha$ IPHAS survey (Drew et al. 2005) and the near-infrared UKIDSS-GPS survey (Lawrence et al. 2007) are near completion, and are already releasing their data products or will start this release soon. In the southern hemisphere, the $\mathrm{H} \alpha \mathrm{VPHAS}+$ and near-infrared VVV ESO public surveys will start observations as soon as their associated instrumentation are available. The scientific exploitation of the survey data will produce an increase of several orders of magnitude of the number of Be stars known in our Galaxy.

All these developments configure a scenario of an exciting and rapidly evolving field of research. In this context, it seems timely for a new major international meeting of researchers on active B stars in the 2009-2011 time frame. Although it is still in the initial discussion stage, actions in this direction have already been undertaken

Juan Fabregat \& Geraldine J. Peters co-chairs of the Working Group

\section{References}

Cameron, C., Saio, H., Kuschnig, R. et al. 2008, ApJ, 685, 489

Domiciano de Souza, A., Kervella, P., Jankov, S. et al. 2003, A\&A (Letters), 407, L47

Drew, J. E., Greimel, R., Irwin, M. J., et al. 2005, MNRAS, 362, 753 
Gies, D. R., Bagnuolo, W. G., Baines, E. K., et al. 2007, ApJ, 654, 527

Lawrence, A., Warren, S. J., Almaini, O., et al. 2007, MNRAS, 379, 1599

Meilland, A., Millour, F., Stee, P., et al. 2007, A\& $A$, 464, 59

Michel, E., Baglin, A., Auvergne, M., et al. 2006, in: M. Fridlund, A. Baglin, J. Lochard \& L. Conroy (eds.), The CoRoT Mission, ESA-SP, 1306, 39

Neiner, C., Hubert, A. M., Frémat, Y., et al. 2003, A\&A, 409, 275

Okazaki, A. T., Owoki, S. P., \& Stefl, S. (eds.) 2007, Active OB-Stars: Laboratories for Stellar and Circumstellar Physics, ASP-CS, 361

Peters, G. J., Gies, D. R., Grundstrom, E. D., \& McSwain, M. V. 2008, ApJ, 686, 1280

Saio, H., Cameron, C., Kuschnig, R., et al. 2007, ApJ, 654, 544

Walker, G. A. H., Kuschnig, R., Matthews, J. M., et al. 2005a, ApJ (Letters), 623, L145

Walker, G. A. H., Kuschnig, R., Matthews, J. M. et al. 2005b, ApJ (Letters), 635, L77 